\title{
Identification of the Oscillator Parameters of the Van der Pol Oscillator Using Wavelet Analysis
}

\author{
N. T. Abdullayev, O. A. Dyshin, D. A. Dadashova \\ Azerbaijan Technical University (Baku), Baku, Azerbaijan \\ Email: a.namik46@mail.ru
}

How to cite this paper: Abdullayev, N.T., Dyshin, O.A. and Dadashova, D.A. (2017) Identification of the Oscillator Parameters of the Van der Pol Oscillator Using Wavelet Analysis. Open Access Library Journal, 4: e3718.

https://doi.org/10.4236/oalib.1103718

Received: June 7, 2017

Accepted: September 19, 2017

Published: September 22, 2017

Copyright $\odot 2017$ by authors and Open Access Library Inc.

This work is licensed under the Creative Commons Attribution International License (CC BY 4.0).

http://creativecommons.org/licenses/by/4.0/

\section{Open Access}

\begin{abstract}
A procedure for determining the current parameters of the oscillator is based on the calculation of the wavelet coefficients of the signal system using fast discrete wavelet transform and application of the differentiation formulas wavelet expansions.
\end{abstract}

\section{Subject Areas \\ Computational Physics}

\section{Keywords}

Oscillator, Range of Power, Wavelet-Transform, Area of Synchronization

\section{Decomposition of a Function into a Wavelet Series}

\subsection{The Definition of the Scale Function and the Basis Wavelet}

Any function $f(t)$ quadratically summable $R=(-\infty, \infty)$ in the space can be expanded at a given level of resolution $j=j_{0}$ in the wavelet series [1] [2]

$$
f(t)=\sum_{k} s_{j_{0}, k} \varphi_{j_{0}, k}(t)+\sum_{j \geq j_{0}} \sum_{k} d_{j, k} \psi_{j, k}(t),
$$

where

$$
\varphi_{j, k}(t)=2^{j / 2} \varphi\left(2^{j} t-k\right), \psi_{j, k}(t)=2^{j / 2} \psi\left(2^{j} t-k\right) .
$$

As introduced $\varphi(t)$ and $\psi(t)$ we will take Daubechies [3] scaling (scale) $\varphi_{M}(t)$ wavelet basis function $\psi_{M}(t)$ and they are defined by the equations:

$$
\varphi(t)=\sqrt{2} \cdot \sum_{k=0}^{2 M-1} h_{k} \varphi(2 t-k), \quad \psi(t)=\sqrt{2} \cdot \sum_{k=0}^{2 M-1} g_{k} \varphi(2 t-k),
$$


where $M$ : positive integer.

Within the framework of multiresolution analysis [4] functions $\varphi_{j, k}(t)$ and $\psi_{j, k}(t)$ serve as a high-frequency and low-frequency filters, RHR, respectively $h_{k}$. The general properties of the scaling functions and wavelet coefficients are uniquely determined. Example of calculation for Daubechies $\left(D^{4}\right)$ wavelet is given in [1].

For Daubechies $h_{k}$ wavelets are real and

$$
s_{j, k}=\left\langle f, \varphi_{j, k}\right\rangle, d_{j, k}=\left\langle f, \psi_{j, k}\right\rangle,
$$

where for any $f_{1}(t)$ and $f_{2}(t)\left(L_{2}(R)\right.$. Here its means that $L_{2}(R)$ space of quadratically summable functions on $R$

$$
\left\langle f_{1}, f_{2}\right\rangle=\int_{R} f_{1}(t) f_{2}(t) \mathrm{d} t .
$$

Using formulas fast wavelet transform

$$
s_{j, n}=\sum_{k=0}^{2 M-1} h_{k} \cdot s_{j-1,2 n+k}, \quad d_{j, n}=\sum_{k=0}^{2 M-1} g_{k} \cdot s_{j-1,2 n+k} .
$$

\subsection{Dependence of Signal Scale on Its Resolution Level}

When time signal $f \in L_{2}(R)$ analysis should choose, the thinnest scale to the subsequent signal synthesis receives it in its original form. We can assume that such a scale is associated with the level of permissions $j=0$. Therefore, the analysis begins with the calculation

$$
s_{0, k}:=\left\langle f, \varphi_{o, k}\right\rangle=\int_{R} f(t) \varphi(t-k) \mathrm{d} t .
$$

These values can be calculated, for example by means of numerical integration. In the case where $f$ the initially set is a discrete array $\{f(k)\}, k \in Z \quad(Z$ : set of integers), just suppose

$$
s_{0, k}:=f(k), k \in Z .
$$

Applying wavelet transform (7), we can now calculate all the factors $s_{j, n}, d_{j, n}$.

Denote the matrix elements of the operator in terms of $T_{S S}^{(n)}\left(j, k ; j, k^{\prime}\right)$, $T_{S D}^{(n)}\left(j, k ; j, k^{\prime}\right), T_{D S}^{(n)}\left(j, k ; j, k^{\prime}\right), T_{D D}^{(n)}\left(j, k ; j, k^{\prime}\right)$. Here

$$
T_{S S}^{(n)}\left(j, k ; j, k^{\prime}\right)=\int_{R} \varphi_{j, k}(t)\left(T^{(n)} \varphi_{j, k^{\prime}}(t)\right) \mathrm{d} t,
$$

replacement of the lower indices $S \rightarrow D(D \rightarrow S)$ in the left side of (9) corresponds to the substitution $\varphi \rightarrow \psi(\psi \rightarrow \varphi)$ of the integral sign on the right side. We denote further $r_{j, l, j, l^{\prime}}^{(n)}=T_{S S}^{(n)}\left(j, l ; j, l^{\prime}\right), \quad r_{k}^{(n)}=T_{S S}^{(n)}(0,0 ; 0, k)$. It's obvious that $r_{j, l, j, l^{\prime}}^{(k)}=r_{j, k \mid k=l-l^{\prime}}^{(k)}$, where $r_{j, k}^{(k)}=r_{j, 0 ; j, k}^{(k)}$.

Elements $r_{j, k}^{(n)}$ of the same level are related

$$
r_{j, k}^{(n)}=2 \sum_{i=0}^{L} \sum_{m=0}^{L} h_{i} \cdot h_{m} \cdot r_{j, 2 k-i+m}^{(n)}, \quad(L=2 M-1),
$$

and the elements adjacent levels-ratio 


$$
r_{j+1, l ; j+1, l^{\prime}}^{(n)}=2^{n} \cdot r_{j, l ; j, l^{\prime}}^{(n)} .
$$

Conditions normalization coefficients $r_{0, k}^{(n)}$ is determined as [1]

$$
\sum_{k=1}^{L} k \cdot r_{0, k}^{(n)}=n ! .
$$

If $j=0 \quad(10)$ for the value of $r_{0, k}^{(n)}$ (the $r_{k}^{(n)}$ ) we obtain the system of equations

$$
r_{k}^{(n)}=2 \sum_{i=0}^{L} \sum_{m=0}^{L} h_{i} \cdot h_{m} \cdot r_{2 k-i+m}^{(n)}, \quad(k=0,1, \cdots, L-2) .
$$

In the domain of the $L$ wavelet coefficients $r_{k}^{(n)}$ in length, they have the symmetry property

$$
r_{k}^{(n)}=(-1)^{k} \cdot r_{-k}^{(n)} \quad(k=1,2, \cdots)
$$

\section{Solution of a System of Linear Algebraic Equations}

\subsection{Operator Matrix Elements of Differentiation the Signal}

Given a $L^{\prime} \geq n \quad\left(L^{\prime}=L-1\right)$ system of linear algebraic Equations (12) - (14) has a unique exact solution $u=\left(r_{0}^{(n)}, r_{1}^{(n)}, \cdots, r_{L-2}^{(n)}\right)$. The matrix elements of the differentiation operator recurrently expressed in terms of matrix elements of the operator of differentiation $T^{(n-1)}=\frac{\mathrm{d}^{n-1}}{\mathrm{~d} t^{n-1}} \quad$ [5].

Solving the system of linear algebraic equations (12) - (14) $n=1$ for all matrix elements $r_{0, l ; 0, l^{\prime}}^{(k)}=\left.r_{k}^{(1)}\right|_{k=l^{\prime}-l}$ can be recovered from the elements.

$T_{S S}^{(1)}\left(j, l ; j, l^{\prime}\right)=r_{j, l, j, l^{\prime}}^{(1)}(j \geq 1)$ using the recurrence relation (11). The remaining matrix elements are $n=1$ defined as follows. By implementing the equivalent of (10) in the $n=1$ expression

$$
T_{S S}^{(1)}\left(j, l ; j, l^{\prime}\right)=2 \sum_{i=0}^{L} \sum_{m=0}^{L} h_{i} \cdot h_{m} \cdot r_{j, 2 l+i ; j, 2 l^{\prime}+m}
$$

replacement indexes $S \rightarrow D$ and respective substitute $h \rightarrow g$, obtain for all $j \geq 0$ presentation

$$
\begin{aligned}
& T_{S D}^{(1)}\left(j, l ; j, l^{\prime}\right)=2 \sum_{i=0}^{L} \sum_{m=0}^{L} h_{i} \cdot g_{m} \cdot r_{j, 2 l+i ; j, 2 l^{\prime}+m}^{(1)}, \\
& T_{D S}^{(1)}\left(j, l ; j, l^{\prime}\right)=2 \sum_{i=0}^{L} \sum_{m=0}^{L} g_{i} \cdot h_{m} \cdot r_{j, 2 l+i ; j, 2 l^{\prime}+m}^{(1)}, \\
& T_{D D}^{(1)}\left(j, l ; j, l^{\prime}\right)=2 \sum_{i=0}^{L} \sum_{m=0}^{L} g_{i} \cdot g_{m} \cdot r_{j, 2 l+i ; j, 2 l^{\prime}+m}^{(1)} .
\end{aligned}
$$

\subsection{Representation of Time Functions in the Form of Series with Wavelet Coefficients}

Let $f(t)$ represented as $g(t)=T^{(n)} f(t)$ a series of wavelet coefficients $\left({ }_{f} s_{j, k} ;{ }_{f} d_{j, k}\right)$ and $\left({ }_{g} s_{j, k} ;{ }_{g} d_{j, k}\right)$. Then 


$$
\begin{aligned}
& { }_{g} S_{j, k}=\sum_{k^{\prime}=0}^{L}\left(T_{S S}^{(n)}\left(j, k ; j, k^{\prime}\right) \cdot{ }_{f} s_{j, k^{\prime}}+T_{S D}^{(n)}\left(j, k ; j, k^{\prime}\right) \cdot{ }_{f} d_{j, k^{\prime}}\right) \\
& { }_{g} d_{j, k}=\sum_{k^{\prime}=0}^{L}\left(T_{D S}^{(n)}\left(j, k ; j, k^{\prime}\right) \cdot{ }_{f} s_{j, k^{\prime}}+T_{D D}^{(n)}\left(j, k ; j, k^{\prime}\right) \cdot{ }_{f} d_{j, k^{\prime}}\right) .
\end{aligned}
$$

The matrix elements are $n=2$ connected to the matrix elements $n=1$ by relations with

$$
\begin{aligned}
& T_{S S}^{(2)}\left(j, k ; j, k_{2}\right)=\sum_{k_{1}=0}^{L}\left(T_{S S}^{(1)}\left(j, k ; j, k_{1}\right) \cdot T_{S S}^{(1)}\left(j, k ; j, k_{2}\right)+T_{S S}^{(1)}\left(j, k ; j, k_{1}\right) \cdot T_{D S}^{(1)}\left(j, k ; j, k_{2}\right)\right), \\
& T_{S D}^{(2)}\left(j, k ; j, k_{2}\right)=\sum_{k^{\prime}=0}^{L}\left(T_{S S}^{(1)}\left(j, k ; j, k_{1}\right) \cdot T_{S D}^{(1)}\left(j, k ; j, k_{2}\right)+T_{S D}^{(1)}\left(j, k ; j, k_{1}\right) \cdot T_{D D}^{(1)}\left(j, k ; j, k_{2}\right)\right), \\
& T_{D S}^{(2)}\left(j, k ; j, k_{2}\right)=\sum_{k^{\prime}=0}^{L}\left(T_{D S}^{(1)}\left(j, k ; j, k_{1}\right) \cdot T_{S S}^{(1)}\left(j, k ; j, k_{2}\right)+T_{D D}^{(1)}\left(j, k ; j, k_{1}\right) \cdot T_{D S}^{(1)}\left(j, k ; j, k_{2}\right)\right), \\
& T_{D D}^{(2)}\left(j, k ; j, k_{2}\right)=\sum_{k^{\prime}=0}^{L}\left(T_{D S}^{(1)}\left(j, k ; j, k_{1}\right) \cdot T_{S D}^{(1)}\left(j, k ; j, k_{2}\right)+T_{D D}^{(1)}\left(j, k ; j, k_{1}\right) \cdot T_{D D}^{(1)}\left(j, k ; j, k_{2}\right)\right) .
\end{aligned}
$$

Replacing in the equation oscillator Van der Pol $x(t), \mathrm{d} x / \mathrm{d} t$ and $\mathrm{d}^{2} x / \mathrm{d} t^{2}$ expansions in the wavelet basis with the carrier. Applying the formula of differentiation (12) and taking $t$ the points $k=0,1, \cdots, L$, we get in the parameter $\lambda$ system of linear algebraic equations type

$$
A \Lambda=b
$$

where $A=\left(a_{0}, a_{1}, \cdots, a_{L}\right)^{\mathrm{T}}$ - the vector of one element $\lambda, \quad b=\left(b_{0}, b_{1}, \cdots, b_{L}\right)^{\mathrm{T}}$ $\mathrm{T}$ : a sign of transposition.

Overdetermined system (19) does not have an exact solution, so instead of an exact solution is organized search of the vector $\Lambda$ that will best meet all the equations of the system (19), i.e., minimize the residual (the difference between the vector $A \Lambda$ and the vector), i.e. $|A \Lambda-b| \rightarrow \min$. Such a solution can be obtained by solving function MATHCAD system.

\section{Advantages of Determining the Parameters of an Oscillator by Solving a System of Equations in the Space of Wavelet Coefficients}

Thus, the calculation of the original signal wavelet coefficients using fast wavelet transform and application of the formulas of differentiation of the discrete wavelet decomposition reduces the problem of determining the oscillator parameters directly to solving a system of linear algebraic equations directly in the space of wavelet coefficients, which significantly increases the speed of calculations in comparison with method of direct and inverse wavelet transforms. This approach has undoubted advantages compared with the numerical $x(t)$ signal by differentiating the approximate mathematical formulas. In addition, if a normally distributed random process is added to the analyzed signal, the formal application of numerical differentiation formulas based on the Newton interpolation polynomials leads to large errors in the evaluation of the derivatives, as shown in [6]. Based wavelet approach provides at least an order of magnitude less than the magnitude of error, and this error can be reduced even further by a 
suitable choice of wavelet basis. The undoubted advantage of wavelet transform is the possibility of eliminating the incorrectness of the operation of numerical differentiation of noisy time series by transitioning into the space of wavelet coefficients.

\section{References}

[1] Dremin, I.M., Ivanov, O.V. and Nechitailo, V.A. (2001) Wavelets and Their Uses. Successes of Physical Sciences, 171, 465-501.

[2] Blatter, K. (2006) Wavelet Analysis. Basic Theory. Translated from English, Technosphere, $272 \mathrm{p}$.

[3] Daubechies, I. (2004) Wavelet Ten Lectures. Moscow Izhevsk, IKI, 163 p.

[4] Mallat, S. (1989) Multiresolution Approximation and Wavelets. Transactions of the American Mathematical Society, 315, 69-88.

[5] Dyshin, O.A. (2008) The Method of Calculating the Matrix of Sustainable Performance in Wavelet Basis Functions of Differential Operators. Proceedings of the Azerbaijan, 27, 76-82.

[6] Koronovsky, A.A., Kurovskaya, M.C. and Khramov, A.E. (2009) Distribution of the Laminar Phases for the Intermittency of Type I in the Presence of Noise. Applied Nonlinear Dynamics, 17, 43-59.

Submit or recommend next manuscript to OALib Journal and we will provide best service for you:

- Publication frequency: Monthly

- 9 subject areas of science, technology and medicine

- Fair and rigorous peer-review system

- Fast publication process

- Article promotion in various social networking sites (LinkedIn, Facebook, Twitter, etc.)

- Maximum dissemination of your research work

Submit Your Paper Online: Click Here to Submit

Or Contact service@oalib.com 